\title{
On leading charmed meson production in $\pi$-nucleon interactions
}

\author{
V.A. Bednyakov \\ Joint Institute for Nuclear Research, Dubna, Russia
}

\begin{abstract}
It is shown that the $D$-meson, whose light quark is the initial-pion valence quark and whose charmed quark is produced in annihilation of valence quarks and has got a large enough momentum, is really a leading meson in reactions like $\pi^{-} p \rightarrow D X$. If such annihilation of valence quarks from initial hadrons is impossible there must be no distinct leading effect.
\end{abstract}

Recently the E769 collaboration [1] has reported confirmation of previously obtained [2] enchanced leading production of $D^{ \pm}$- and $D^{* \pm}$-mesons in $250 \mathrm{GeV}$ $\pi^{ \pm}$-nucleon interaction. A leading charmed meson is considered to be one with the longitudinal momentum fraction $x_{F}>0$, whose light quark (or anti-quark) is of the same type as one of the quarks in the beam particle. At large $x_{F}$ significant asymmetry was found:

$$
A\left(x_{F}\right) \equiv \frac{\sigma(\text { leading })-\sigma(\text { non-leading })}{\sigma(\text { leading })+\sigma(\text { non-leading })} .
$$

Such asymmetry for the production of charmed hadrons is not expected in perturbative quantum chromodynamics.

Some years ago a simple non-perturbative mechanism of leading charmed mesons production was considered [3] for data analysis of CERN experiment on $D$-mesons production in $\pi^{-} p$-collisions 41 . It was demonstrated that presence

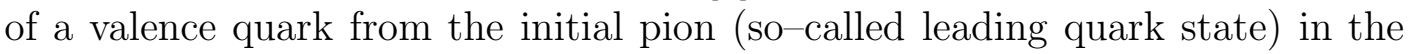
final charmed meson is a necessary but insufficient condition for the meson to be a leading one. Actually, those $D$ are leading mesons whose light quarks are valence quarks of the pion and charmed quarks are produced in annihilation of valence quarks and carry a large momentum $x_{c}$.

The leading effect is a characteristic property of inclusive production of charmed hadrons [5]. A hadron $H$ produced in the reaction $a+b \rightarrow H+\ldots$ and carrying the largest portion of the momentum, $p_{H}=O(\sqrt{s} / 2)$, is regarded as a leading hadron. The corresponding momentum spectrum $d N / d x_{F}$ usually parametrised in the form $\left(1-x_{F}\right)^{n}$ at a large Feynman variable $x_{F}=\frac{2}{\sqrt{s}} P_{\|}$is "hard" for leading hadrons $(0<n \lesssim 3)$ and "soft" for non-leading ones $(n \gtrsim 5)$.

In the quark-parton approach the leading charmed meson $H$ is a result of recombination of the spectator valence quark $q_{v}$ with the charmed quark produced in a parton subprocess. Owing to the large momentum of the valence quark $x_{v} H$ turns to be a leading meson, its momentum is large enough $x_{H}=$ $x_{v}+x_{c}>x_{v}$. 
From this point of view $D^{-}(d \bar{c})$ and $D^{\circ}(\bar{u} c)$ directly produced in the reaction $\pi^{-}(d \bar{u})+p \rightarrow D(d \bar{c} ; \bar{u} c)+X$ must be both leading mesons, i.e. yields of $D^{-}(d \bar{c})$ and $D^{\circ}(\bar{u} c)$ have to be practically the same at large momentum (say, $x_{F}>0.5$ ).

On the other hand, let us assume for a moment that hadrons consist of valence quarks alone. This picture takes place, for instance, in deep inelastic phenomena at quite large $x_{F}$, when all non-singlet parton distribution functions vanish.

In this case $D^{\circ}(\bar{u} c)$-mesons can by no means result form the reaction $\pi^{-}(d \bar{u})+$ $p($ uud $) \rightarrow D+X$ because there is no parton subprocess which can ensure $c$ quark creation. On the other hand, the $\bar{c}-$ quark appears due to valence quarks annihilation $\bar{u}_{v}^{\pi} u_{v}^{p} \rightarrow c \bar{c}$, providing the $D^{-}(d \bar{c})$-meson in the final state. It is clear that some difference in $\pi^{-}$-nucleon production of leading $D^{\circ}(\bar{u} c)$ and $D^{-}(d \bar{c})$-meson has to take place at sufficiently large $x_{F}$. To demonstrate this feature quantitatively let us follow briefly the work [3].

The invariant differential cross section for the process $\pi^{-} p \rightarrow D X$ in the centre-of-mass system at the energy $\sqrt{s}$ and $x_{F}>0$ can be written down in the form [6]:

$$
x^{*} \frac{d \sigma}{d x d p_{T}^{2}}=\exp \left\{-2 p_{T}^{2} / \sqrt{s}\right\} \int R\left(x_{s p}, x_{c} ; x\right) \frac{d x_{s p}}{x_{s p}} \frac{d x_{c}}{x_{c}^{*}}\left\{\frac{x_{c}^{*} x_{s p} d \sigma}{d x_{s p} d x_{c} d p_{T}^{2}}\right\} .
$$

Here $x \equiv x_{F}, x_{s p}, x_{c}$ are the Feynman variables of $D^{-}\left(D^{\circ}\right)$-meson, spectator $d(\bar{u})$ - and produced $\bar{c}(c)$-quark; $x^{*}=2 E_{D} / \sqrt{s}, x_{c}^{*}=2 E_{c} / \sqrt{s}$.

The phenomenological recombination function [6], [7] $R\left(x_{s p}, x_{c} ; x\right) \sim \delta(x-$ $x_{s p}-x_{c}$ ) provides a probability of producing a $D^{-}\left(D^{\circ}\right)$-meson (with the momentum $x$ ) by means of a $d(\bar{u})$-quark $\left(x_{s p}\right)$ and a $\bar{c}(c)$-quark $\left(x_{c}\right)$.

The probability of existence of spectator $d(\bar{u})$-quark and charmed $\bar{c}(c)$-quark is determined by the expression:

$$
\frac{x_{c}^{*} x_{s p} d \sigma}{d x_{s p} d x_{c} d p_{T}^{2}}=x_{s p} \int d x_{L} d x_{R} \sum_{i=q, \bar{q}, g} f_{d(\bar{u}) i}^{\pi}\left(x_{s p}, x_{L}\right) f_{\bar{i}}^{p}\left(x_{R}\right) \frac{x_{c}^{*} d \sigma}{d x_{c} d p_{T}^{2}} .
$$

Here $\frac{x_{c}^{*} d \sigma}{d x_{c} d p_{T}^{2}}$ is the quantum-chromodynamics cross section for the charm production parton subprocess $i \bar{i} \rightarrow c \bar{c}[\mathbb{Q}$. The single-particle proton distribution functions, $f_{i}^{p}\left(x_{R}\right)$, are extracted from deep inelastic lepton-proton scattering [9]. The analytical form of two-particle pion distribution functions, $f_{v i}^{\pi}\left(x_{s p}, x_{L}\right)$, is given in the statistical parton model [6], [10]. The free parameters of these analytical forms can be fixed via comparison with the data.

It is clear from relation (3) that the above-mentioned difference in yields of $D^{\circ}(\bar{u} c)$ and $D^{-}(d \bar{c})$ - mesons mainly arises due to different contributions of distribution functions: $\sum f_{v i}^{\pi} \cdot f_{\bar{i}}^{p}$.

For a $D^{\circ}$-meson the sum is

$$
\sum D^{\circ}=f_{v v}^{\pi} \cdot f_{s}^{p}+f_{v s}^{\pi} \cdot\left(3 f_{v}^{p}+6 f_{s}^{p}\right) .
$$


For a $D^{-}$-meson we have

$$
\sum D^{-}=f_{v v}^{\pi} \cdot f_{s}^{p}+f_{v s}^{\pi} \cdot\left(3 f_{v}^{p}+6 f_{s}^{p}\right)+2 f_{v v}^{\pi} \cdot f_{v}^{p}=\sum D^{\circ}+2 f_{v v}^{\pi} \cdot f_{v}^{p},
$$

where index $v$ corresponds to valence quarks and $s$ to sea quark. For simplicity flavour symmetric distributions were used and the gluon contribution was omitted.

Therefore the total momentum spectrum of $D^{-}$and $D^{\circ}-$ meson production in $\pi^{-} p$-collisions can be put down in the form

$$
\frac{d \sigma}{d x}\left(D^{-}+D^{\circ}\right)=2 \frac{d \sigma}{d x}\left(D^{\circ}\right)+\frac{d \sigma}{d x}(v) .
$$

This formula was used for fixing distribution functions $f_{v i}^{\pi}$ by means of comparison with the data on leading $D$-meson production in $\pi^{-} p$-collisions at $\sqrt{s}=26 \mathrm{GeV}$. 1 .

It was obtained that the "valence" component, $\frac{d \sigma}{d x}(v)$, due to "hard" shape of valence distributions, ensured the non-vanishing total spectrum for $x_{F} \gtrsim 0.5$. At low $x_{F}$ the total spectrum was saturated by the other component $-\frac{d \sigma}{d x}\left(D^{\circ}\right)$.

The term $\frac{d \sigma}{d x}(v)$ makes no contribution to the spectrum of $D^{\circ}$-mesons (see formula (乐), therefore the yield of neutral $D^{\circ}$-mesons at large $x_{F}$ is small enough.

Figure 1 shows the ratio:

$$
R\left(x_{F}\right)=\frac{\frac{d \sigma}{d x}\left(\pi^{-} p \rightarrow D^{\circ} X\right)}{\frac{d \sigma}{d x}\left(\pi^{-} p \rightarrow D^{-} X\right)}
$$

which quantitatively illustrates the suppression of the $D^{\circ}$ yield as comparied with the $D^{-}$one. The experimental points are recalculated from combined data on asymmetry $A$ (1) measured on nuclei [1]. The curves obtained in paper [3] and considered as a predictions successfully fit the new data [1].

Figure 2 shows two curves for asymmetry $A$ (1), calculated on the basis of the ratio (7). The curves also describe the data well.

Thus it is demonstrated that presence of a valence quark from the initial hadron (as a spectator) in the final charmed meson is a necessary but insufficient condition for the meson to have a "hard" momentum spectrum (i.e. to be a leading meson).

Actually, the $D$-meson is a "real" leading meson whose light quark is a spectator valence quark and charmed quark (anti-quark) is produced in annihilation of valence quarks from initial hadrons.

In addition, it is easy to construct relations like (17) for reactions similar to $\pi^{-} p \rightarrow D X$. Thus we have for $x_{F}>0.5$ (denominators show the leading mesons):

$$
\frac{\sigma\left(\pi^{+} n \rightarrow D^{+} X\right)}{\sigma\left(\pi^{+} n \rightarrow \bar{D}^{\circ} X\right)}=\frac{\sigma\left(\pi^{+} \bar{p} \rightarrow \bar{D}^{\circ} X\right)}{\sigma\left(\pi^{+} \bar{p} \rightarrow D^{+} X\right)}=\frac{\sigma\left(\pi^{-} \bar{n} \rightarrow D^{-} X\right)}{\sigma\left(\pi^{-} \bar{n} \rightarrow D^{\circ} X\right)}=R\left(x_{F}\right) ;
$$




$$
\begin{gathered}
\frac{\sigma\left(K^{-} p \rightarrow \bar{D}^{\circ} X\right)}{\sigma\left(K^{-} p \rightarrow D_{s}^{-} X\right)}=\frac{\sigma\left(K^{+} \bar{p} \rightarrow D^{\circ} X\right)}{\sigma\left(K^{+} \bar{p} \rightarrow D_{s}^{+} X\right)}=R\left(x_{F}\right) \\
\frac{\sigma\left(\pi^{-} \bar{p} \rightarrow D^{-} X\right)}{\sigma\left(\pi^{-} \bar{p} \rightarrow D^{\circ} X\right)}=\frac{\sigma\left(\pi^{+} p \rightarrow D^{+} X\right)}{\sigma\left(\pi^{+} p \rightarrow \bar{D}^{\circ} X\right)}=\frac{\sigma\left(\pi^{-} n \rightarrow D^{\circ} X\right)}{\sigma\left(\pi^{-} n \rightarrow D^{-} X\right)}=2 R\left(x_{F}\right) ; \\
\frac{\sigma\left(\pi^{+} \bar{n} \rightarrow \bar{D}^{\circ} X\right)}{\sigma\left(\pi^{+} \bar{n} \rightarrow D^{+} X\right)}=\frac{\sigma\left(K^{-} n \rightarrow \bar{D}^{\circ} X\right)}{\sigma\left(K^{-} n \rightarrow D_{s}^{-} X\right)}=\frac{\sigma\left(K^{+} \bar{n} \rightarrow \bar{D}^{\circ} X\right)}{\sigma\left(K^{+} \bar{n} \rightarrow D_{s}^{+} X\right)}=2 R\left(x_{F}\right) .
\end{gathered}
$$

\section{References}

[1] E769 Coll. G.A.Alves et al, Fermilab-Pub-93/310-E, Fermilab, October 1993 (Submitted to Phys.Rev.Lett).

[2] ACCMOR Coll. S. Barlag et al Z.Phys. C 49, (1991) 555; WA82 Coll. M. Adamovich et al Phys.Lett. B305, (1993) 402; E769 Coll. G.A.Alves et al, Phys.Rev.Lett. 69 (1992) 3147.

[3] Bednyakov V.A. "The fast charm quark and leading $D^{-}$-mesons in $\pi^{-} p$ collisions", Preprint JINR, E2-86-789, Dubna, 1986. (unpublished.)

[4] Aguilar-Benitez M. et al. Phys.Lett., 1985, B161, p.400; Z.Phys.C, 1986, 31, p. 491; Begalli M. et al., Yad. Fiz. 1986, 44, p. 998.

[5] Basile M. et al., Nuovo Cim., 1981, A66, p. 129; Kerman A., Van Dalen G., Phys. Rep. 1984, 106, p. 209.

[6] Kartvelishvili V.G., Likhoded A.K., Slabospitsky S.R., Yad. Fiz. 1981, 33, p. 832; Likhoded A.K., Slabospitsky S.R., Suslov M.V., Yad. Fiz. 1983, 38, p. 727; Bednyakov V.A., Bunyatov S.A., Isaev P.S. JINR Communication, P2-86-739, Dubna, 1986.

[7] Takasugi E. et al., Phys. Rev. 1979, D20, p. 211.

[8] Glück M. et al., Phys. Rev. 1978, D17, p. 2324.

[9] Zlatev I.S. et al. Yad. Fiz. 1982, 35, p. 454.

[10] Bednyakov V.A., Isaev P.S., Kovalenko S.G. Yad. Fiz. 1984, 40, p. 1312.

\section{Figure Captions}

Fig. 1. $D^{\circ}-$ to $-D^{-}$yield ratios (7) for $\pi^{-} p$-collisions (lower curve) and $\pi^{-} n-$ collisions (upper curve). The points are recalculated from the data on asymmetry $A$ [1].

Fig. 2. Asymmetry $A(1)$ on the proton target (upper curve) and the neutron target (lower curve) calculated on the basis of the ratio (17). The data from ref. [回]. 
This figure "fig1-1.png" is available in "png" format from: http://arxiv.org/ps/hep-ph/9403270v1 